\section{Biology of soft tissue wound healing and regeneration - Consensus Report of Group 1 of the 10th European Workshop on Periodontology}

Hämmerle CHF, Giannobile WV. Biology of soft tissue wound healing and regeneration. Consensus Report of Group 1 of the 10th European Workshop on Periodontology. J Clin Periodontol 2014; 41 (Suppl. 15): S1-S5. doi: 10.1111/jcpe.12221.

\begin{abstract}
Background: The scope of this consensus was to review the biological processes of soft tissue wound healing in the oral cavity and to histologically evaluate soft tissue healing in clinical and pre-clinical models.

Aims: To review the current knowledge regarding the biological processes of soft tissue wound healing at teeth, implants and on the edentulous ridge. Furthermore, to review soft tissue wound healing at these sites, when using barrier membranes, growth and differentiation factors and soft tissue substitutes.

Collection of data: Searches of the literature with respect to recessions at teeth and soft tissue deficiencies at implants, augmentation of the area of keratinized tissue and soft tissue volume were conducted. The available evidence was collected, categorized and summarized.

Fundamental principles of oral soft tissue wound healing: Oral mucosal and skin wound healing follow a similar pattern of the four phases of haemostasis, inflammation, proliferation and maturation/matrix remodelling. The soft connective tissue determines the characteristics of the overlaying oral epithelium. Within 7-14 days, epithelial healing of surgical wounds at teeth is completed. Soft tissue healing following surgery at implants requires 6-8 weeks for maturation. The resulting tissue resembles scar tissue. Well-designed pre-clinical studies providing histological data have been reported describing soft tissue wound healing, when using barrier membranes, growth and differentiation factors and soft tissue substitutes. Few controlled clinical studies with low numbers of patients are available for some of the treatments reviewed at teeth. Whereas, histological new attachment has been demonstrated in pre-clinical studies resulting from some of the treatments reviewed, human histological data commonly report a lack of new attachment but rather long junctional epithelial attachment and connective tissue adhesion. Regarding soft tissue healing at implants human data are very scarce.

Conclusions: Oral soft tissue healing at teeth, implants and the edentulous ridge follows the same phases as skin wound healing. Histological studies in humans have not reported new attachment formation at teeth for the indications studied. Human histological data of soft tissue wound healing at implants are limited.
\end{abstract}

Christoph H. F. Hämmerle ${ }^{1}$ and William V. Giannobile ${ }^{2}$ on behalf of Working Group 1 of the European Workshop on Periodontology*

${ }^{1}$ University of Zurich, Zurich, Switzerland;

${ }^{2}$ University of Michigan, Ann Arbor, MI, USA
Key words: barrier membranes; growth and differentiation factors; human histology; oral soft tissue; periodontal/peri-implant wound healing; pre-clinical studies; scaffolds; soft tissue substitutes

Accepted for publication 12 November 2013 
Clinical recommendations: The use of barriers membranes, growth and differentiation factors and soft tissue substitutes for the treatment of localized gingival/ mucosal recessions, insufficient amount of keratinized tissue and insufficient soft tissue volume is at a developing stage.
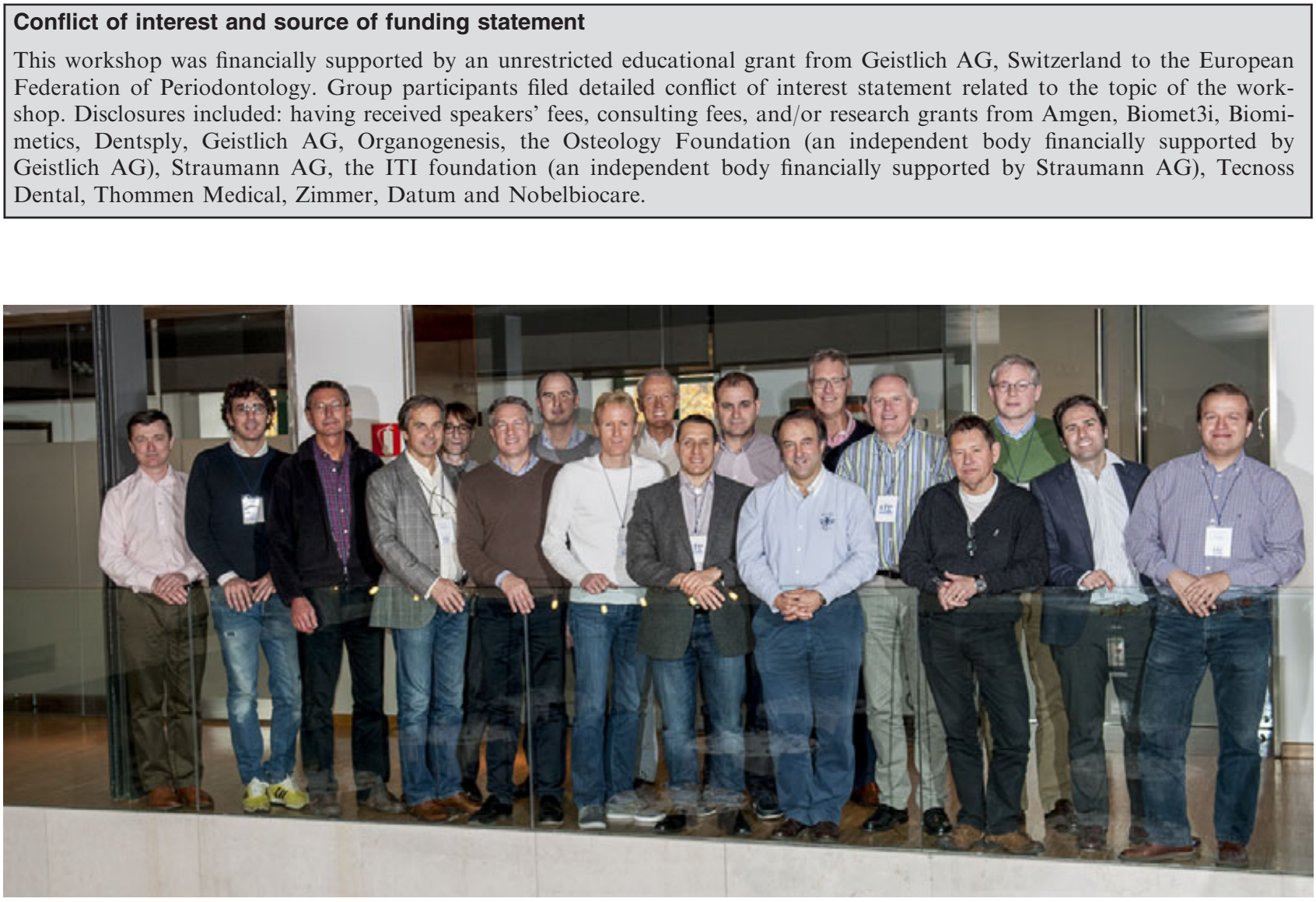

*Group 1 participants: Ingemar Abrahamsson, Juan Blanco, Dieter Bosshardt, Christer Dahlin, Nikos Donos, Jan Eirik Ellingsen, Stefan Fickl, Reinhard Gruber, Christoph Goerlach, Javier Nuñez, Joerg Meyle, Frank Schwarz, Anton Sculean, Andreas Stavropoulos, Hendrik Terheyden, Fabio Vignoletti, Franz Weber

This consensus group evaluated in the first review emphasis the current evidence relating to the basic biological concepts of the oral wound healing response. As a part of the second review, a particular emphasis on early stage investigations of newly developed biological agents for periodontal soft tissue regeneration including barrier scaffolding matrices, biological agents (such as enamel matrix derivative, platelet-derived growth factor) and soft tissue substitutes was evaluated. The second review focused on pre-clinical and clinical studies with the requirement of histological biopsy material of the wound repair process.

The scope of Workshop Group 1, focused on two key areas: (i) soft tissue wound healing around teeth and dental implants (Sculean et al. 2014) and (ii) soft tissue wound healing at teeth, dental implants and the edentulous ridge with barrier membranes, growth and differentiation factors and soft tissue substitutes (Vignoletti et al. 2014).

\section{Soft Tissue Wound Healing Around Teeth and Dental Implants (Sculean et al. 2014)}

The narrative review that was prepared by working group la focused on evaluating the wound healing concept in three key areas: Basic oral biological principles, soft tissue healing around teeth and soft tissue repair around dental implants.

\section{Summary of key points of the review:}

1 Oral mucosal and skin wound healing follows a similar pattern of the four phases haemostasis, inflammation, proliferation and maturation/matrix remodelling.

2 Infection control is important for undisturbed oral wound healing and is a prerequisite for the transition of the inflammatory to the proliferative phase.

3 Granulation tissue derived from the periodontal ligament induces epithelial cells to form a keratinized masticatory gingiva.

4 The soft connective tissue determines the characteristics of the overlaying oral epithelium. 
5 Epithelial healing around teeth following non-surgical and surgical periodontal therapy is completed after 7-14 days (Novaes et al. 1969, Stahl et al. 1971, Waerhaug 1978).

6 Functional stability between the denuded root and soft tissue is achieved approximately 14 days after surgery (Hiatt et al. 1968).

7 The formation of the biological width and maturation of the barrier function around transmucosal implants requires 6-8 weeks of healing (Berglundh et al. 2007, Schwarz et al. 2013, Tomasi et al. 2013).

8 The established peri-implant soft connective tissue resembles a scar tissue in composition, fibre orientation and vasculature.

9 The peri-implant junctional epithelium may reach a greater final length under certain conditions such as implants placed into fresh extraction sockets versus conventional implant procedures in healed sites.

\section{Recommendations for future research}

Strategies for future research investigation to advance the field of oral wound healing around teeth and implants included the following target areas recommended by the expert review panel:

- Need more information on the inductive influences of the connective tissue environment on tissue neogenesis. Study at the cellular and matrix levels could be important in determining environmental cues. Cell: cell interactions (e.g. epithelial-mesenchymal influences) may instruct cell fate and determination. Identification of the cytokine, chemokine and intercellular signalling networks that are involved in the wound healing process may better inform on regenerative and reparative approaches. The study of existing and newly discovered growth and differentiation factors may lead to new information on the repair and regeneration of oral tissues.

Evidence gained from genetic models (i.e. transgenic and gene deletion) relevant to skin morphogenesis and regeneration may be instructive and translate to the oral wound healing situation. More study is warranted to examine the oral wound healing response in the face of the microbial challenge and local salivary molecules that may influence epigenetic changes of oral epithelia.

- To identify host and immunological cellular and soluble factors that regulate the healing process during periodontal and periimplant wound repair. Determine what genetic biological phenotypes may regulate an exacerbated healing responses depending on immunological profiles. It would also be valuable to study ageing on oral soft tissue wound healing responses on cellular senescence and telomere length on stem cell progenitors contributing to the reparative process.

- To investigate the mechanisms of connective tissue graft (CTG) integration, re-vascularization and re-epithelialization as it relates to tissue remodelling (increases or decreases in tissue volume and quality) in different soft tissue biotype situations.

- To identify the basic mechanisms and relevance of scar-like periimplant connective tissue and epithelial interface. To determine the mechanisms of connective tissue adhesion to the implant material surface. The field would be advanced to better understand the biological consequences of implant/abutment biomaterial surface characteristics on the influence of biological width formation. The role of implant biomaterial surface characteristics on dental biofilm formation and proliferation should be considered.

\section{Soft Tissue Wound Healing at Teeth, Dental Implants and the Edentulous Ridge When Using Barrier Membranes, Growth and Differentiation Factors and Soft Tissue Substitutes (Vignoletti et al. 2014)}

The narrative review that was prepared by working group $1 \mathrm{~b}$ focused on evaluating the histological results of the application of scaffolds, cells and biologicals on soft tissue wound healing at teeth, dental implants and the edentulous ridge.
Data were collected from preclinical experiments and from patient treatments reporting histological outcomes. Clinical studies, regardless of the evidence level provided, not reporting histological outcomes are not included in this review. As a consequence a large body of data derived from well-designed clinical studies are not included in this review.

The level of evidence available for this review was mostly limited to well-designed pre-clinical studies and to clinical case series or case reports. Few controlled clinical studies with low numbers of patients were available for some of the treatments reviewed. The studies available were mostly of short duration not exceeding 6 months. The studies available for evaluation were primarily associated with teeth. There was limited information available on soft tissue augmentation around implants.

\section{Recessions at teeth and soft tissue deficiencies at dental implants}

Regarding recessions at teeth and soft tissue deficiencies at dental implants the following therapeutic approaches were evaluated: barrier membranes, growth and differentiation factors and soft tissue substitutes.

Indications for the treatment of recessions at teeth and soft tissue deficiencies at dental implants include aesthetic patient desires, root hypersensitivity and difficulty to perform plaque control. When evaluating the treatment approaches dealt with in this review, the coronally advanced flap (CAF) with and without a CTG was used as a control.

Available human histological data were often retrieved following treatment of hopeless teeth, very advanced or experimentally created defects. These extreme situations are far from the expected clinical use and, therefore, are suboptimal examples to assess the biological potential of these new technologies.

\section{Barrier membranes}

When barrier membranes were used at teeth in addition to CAF, increased amounts of new cementum, connective tissue attachment and reduced length of the junctional epithelium were observed. No significant differences were found related 
to these parameters between resorbable and non-resorbable membranes.

The histological data revealed no adverse reactions resulting from the use of these materials.

The clinical differences between CAF with/without membranes were small in pre-clinical studies providing histology.

\section{Growth and differentiation factors}

The reason to introduce growth and differentiation factors is to induce new attachment formation in addition to the clinical coverage of the recession defect.

Very little human histological data are available on the use of growth and differentiation factors.

The factors reported in the review encompassed Enamel Matrix Derivative (EMD), Platelet-Derived Growth Factor (PDGF) and Platelet Rich Plasma (PRP).

The potential of EMD to promote new attachment has consistently been demonstrated in pre-clinical histological studies. Pre-clinical histological data are lacking comparing EMD to the standard of care. Histological data from humans, however, have failed to confirm new attachment formation. Favourable clinical data were reported in some of these human histological studies.

In one histological study in humans PDGF (CAF + PDGF) exhibited new attachment formation in contrast to the control group (CAF + CTG). The clinical comparison in this study favoured the controls regarding recession depth reduction and mean root coverage.

In one pre-clinical histological study comparing $\mathrm{CAF}+\mathrm{CTG}+$ PRP (test) versus the standard of care CAF + CTG, the test group showed more new attachment formation than the control group. When comparing the two procedures, the residual recession was similar in this pre-clinical study. No human histological datum is available.

\section{Soft tissue substitutes}

The reason to introduce soft tissue substitutes is to avoid the harvesting procedure and the concomitant morbidity associated with autogenous soft tissue grafts, which presently are the standard of care.

The following materials have been histologically evaluated: acellu- lar dermal matrix graft (ADMG), collagen matrices (CM), human fibroblast-derived dermal substitute (HF-DDS), and human skin equivalents [bilayered cell therapy (BCT)].

In three pre-clinical studies ADMG or CM have shown histological results regarding the tissuetooth interface similar to the standard of care CAF + CTG and CAF alone, respectively. Human histological data have mostly shown epithelial attachment and connective tissue adhesion with an absence of adverse reactions. Successful recession coverage was reported in some of these human histological studies.

In one pre-clinical study with dental implants the histological results for $\mathrm{CAF}+\mathrm{CM}$ revealed a tissue implant interfaces similar to CAF + CTG or CAF alone consisting of epithelial attachment and connective tissue adhesion. The amount of coverage of the soft tissue deficiencies was similar for the three procedures, however, both experimental groups improved tissue thickness when compared with CAF alone. No human histological datum is available.

Presently, there are no histological data available for HF-DDS and BCT.

\section{Augmentation of keratinized tissue}

The standard of care for increasing the zone of keratinized tissue is the apically positioned flap (APF) with an autogenous graft with/without epithelium, that is free gingival graft (FGG) or CTG.

\section{Soft tissue substitutes}

The following materials have been histologically evaluated: ADMG, CM and human skin equivalents (BCT).

Generally speaking, there is little histological data assessing these materials in this indication. The histological pre-clinical and clinical data available demonstrate these materials to be well integrated into the host connective tissues without adverse reactions.

In one pre-clinical study similar histological appearance was described for the increased zone of keratinized tissue comparing $\mathrm{APF}+\mathrm{CM}$ with APF in edentulous areas of the ridge. The clinical results of this histological study have shown similar and large amounts of gain of keratinized tissue.
Another pre-clinical study compared $\mathrm{APF}+\mathrm{CM}$ to $\mathrm{APF}$ for increasing the zone of keratinized tissue. A tissue-tooth interface was found consisting of epithelial attachment and connective tissue adhesion similar in both groups. The gain in keratinized tissue was similar but small in both groups.

In one human study comparing $\mathrm{APF}+\mathrm{CM}$ from a different source and $\mathrm{APF}+\mathrm{FGG}$, a good integration and keratinization of the CM was found. The clinical results, however, reported smaller amounts of gain of keratinized tissue compared with the controls.

ADMG was tested in a pre-clinical and two human studies for augmentation of keratinized tissue at teeth in combination with APF. Histology revealed good tissue integration and a small gain of keratinized tissue associated with the ADMG.

In one clinical case aiming at increasing the zone of keratinized tissue at an implant site retrieval of a histological sample revealed inflammation and degeneration of the ADMG.

BCT was tested in one human study together with an APF against $\mathrm{APF}+\mathrm{FGG}$. Histologically good integration and keratinization were found for both groups investigated. The FGG group revealed more gain of keratinized tissue.

\section{Augmentation of insufficient soft tissue volume}

The standard of care for increasing the volume of the soft tissue is the transplantation of an autogenous graft with/without epithelium, that is CTG or FGG.

\section{Soft tissue substitutes}

A modified xenogenic collagen matrix (mCM) has been histologically evaluated for soft tissue augmentation and compared with the CTG in one pre-clinical study. The histological analysis demonstrated similar integration into the surrounding tissues. Concomitant clinical measurements of volume gain demonstrated results similar to the one obtained with the CTG.

\section{Implications for Future Research}

- As histological assessments may not be reliable to estimate the 
increase/change in soft tissue thickness, these studies should consider different methodologies/ technologies (e.g. 3D digital imaging analysis).

- There is a need to properly define valid and reproducible pre-clinical models for the assessment of soft tissue regeneration procedures around teeth and implants.

- Future pre-clinical studies should be adequately powered and consider primary outcomes that are relevant for clinical situations (e.g. use of non-histological surrogate measures that can be measured clinically).

- Success criteria associated with soft tissue augmentation around teeth and implants may differ and need to be defined separately.

- Future studies should elaborate on potential indications and the clinical relevance of new soft tissue augmentation procedures and long-term effects.

- Patient-centred/reported outcomes should be an integral part of the evaluation of new biotechnologies when compared with current standard procedures.

- Safety analyses and reporting should, in addition to local (e.g. clinical and histological) signs of potential immunological responses, also consider assessment of antibody levels formed in response to xenogenic- or allogenic-derived biomaterials.

- Health economics should be considered in the assessment of interventions and the introduction of new technologies.

\section{Clinical Relevance}

Manipulations of soft tissues as therapeutic steps associated with teeth and implants are common clinical procedures. An understanding of the biological process of wound healing helps to better

\section{Clinical Consequences}

- The use of barriers membranes, growth and differentiation factors and soft tissue substitutes for the treatment of localized gingival/ mucosal recessions, insufficient amount of keratinized tissue and insufficient soft tissue volume is at a developing stage.

- The conduct of pre-clinical studies is advantageous to preselect potential candidate materials that can be safely used in early stage clinical trials.

- There continues to be a need for well-designed, randomized, parallel-arm-controlled clinical trials to further evaluate the efficacy of new technologies for the treatment of localized gingival/mucosal recessions, limited zones of keratinized tissue and insufficient soft tissue volume. The potential influence of relevant confounding clinical factors (e.g. flap design, suturing techniques) should also be carefully addressed.

\section{References}

Berglundh, T., Abrahamsson, I., Welander, M., Lang, N. P. \& Lindhe, J. (2007) Morphogenesis of the peri-implant mucosa: an experimental study in dogs. Clinical Oral Implants Research 18, 1-8. doi:10.1111/j.1600-0501.2006. 01380.x.

Hiatt, W. H., Stallard, R. E., Butler, E. D. \& Badgett, B. (1968) Repair following mucoperiosteal flap surgery with full gingival retention. Journal of Periodontology 39, 11-16. doi:10. 1902/jop.1968.39.1.11.

Novaes, A. B., Kon, S., Ruben, M. P. \& Goldman, H. M. (1969) Visualization of the micro- vascularization of the healing periodontal wound. 3. Gingivectomy. Journal of Periodontology 40, 359-371. doi:10.1902/jop.1969.40.6. 359.

Schwarz, F., Mihatovic, I., Becker, J., Bormann, K. H., Keeve, P. L. \& Friedmann, A. (2013) Histological evaluation of different abutments in the posterior maxilla and mandible: an experimental study in humans. Journal of Clinical Periodontology 40, 807-815. doi:10.1111/ jcpe. 12115 .

Sculean, A., Gruber, R. \& Bosshardt, D. D. (2014) Soft tissue wound healing around teeth and dental implants. Journal of Clinical Periodontology 41, accepted.

Stahl, S. S., Weiner, J. M., Benjamin, S. \& Yamada, L. (1971) Soft tissue healing following curettage and root planing. Journal of Periodontology 42, 678-684. doi:10.1902/jop.1971. 42.11.678.

Tomasi, C., Tessarolo, F., Caola, I., Wennstrom, J., Nollo, G. \& Berglundh, T. (2013) Morphogenesis of peri-implant mucosa revisited: an experimental study in humans. Clinical Oral Implants Research [Epub ahead of print]. doi:10.1111/clr.12223.

Vignoletti, F., Nunez, J. \& Sanz, M. (2014) Soft tissue wound healing at teeth, dental implants and the edentulous ridge with scaffolds, cells and biologicals. Journal of Clinical Periodontology 41, accepted.

Waerhaug, J. (1978) Healing of the dento-epithelial junction following subgingival plaque control. I. As observed in human biopsy material. Journal of Periodontology 49, 1-8. doi:10.1902/ jop.1978.49.1.1.

\section{Address:}

Christoph H.F. Hämmerle

University of Zürich

Clinic of Fixed and Removable

Prosthodontics and Dental Material Science

Plattenstrasse 11

CH-8032 Zurich, Switzerland

E-mail: christoph.hammerle@zzm.uzh.ch

understand the possibilities and limitations of such clinical preventive or corrective interventions. A range of different products are available aimed at improving the conditions of the soft tissues at teeth, implants and the edentulous ridge. These pro- ducts encompass scaffolds, cells and biologicals. Histolocial evidence from human studies provides valuable guidelines for clinical application of these products. 